\title{
Raw Data
}

National Cancer Institute

\section{Source}

National Cancer Institute. Raw Data. NCI Thesaurus. Code C142663.

The original information, collected from the primary source. 